\title{
External validation of Liaoning score for predicting esophageal varices in liver cirrhosis: a Chinese multicenter cross-sectional study
}

\author{
Qianqian Li ${ }^{1 \#}$, Yunhai $\mathrm{Wu}^{2 *}$, Qiang Zhu ${ }^{3 \#}$, Fanping Meng ${ }^{4 *}, \mathrm{Su} \mathrm{Lin}^{5}$, Bang Liu ${ }^{6}, \mathrm{Bimin} \mathrm{Li}^{7}$, \\ Shanhong Tang ${ }^{8}$, Yida Yang ${ }^{9}$, Yiling $\mathrm{Li}^{10}$, Shanshan Yuan ${ }^{11}$, Yu Chen ${ }^{12}$, Xingshun Qi ${ }^{1}$ \\ ${ }^{1}$ Department of Gastroenterology, General Hospital of Northern Theater Command, Shenyang 110840, China; ${ }^{2}$ Department of Critical Care \\ Medicine, The Sixth People's Hospital of Shenyang, Shenyang 110006, China; ${ }^{3}$ Department of Gastroenterology, Shandong Provincial Hospital \\ Affiliated to Shandong University, Jinan 250021, China; ${ }^{4}$ Department for Biological Therapy, The Fifth Medical Center of PLA General Hospital, \\ Beijing 100039, China; ${ }^{5}$ Liver Research Center, The First Affiliated Hospital of Fujian Medical University, Fuzhou 350005, China; ${ }^{6}$ Department of \\ Hepatobiliary Disease, 900 Hospital of the Joint Logistics Team (formerly called Fuzhou General Hospital), Fuzhou 350025, China; ${ }^{7}$ Department \\ of Gastroenterology, The First Affiliated Hospital of Nanchang University, Nanchang 330006, China; ${ }^{8}$ Department of Gastroenterology, General \\ Hospital of Western Theater Command, Chengdu 610083, China; ${ }^{9}$ State Key Laboratory for Diagnosis and Treatment of Infectious Diseases, \\ Collaborative Innovation Center for Diagnosis and Treatment of Infectious Diseases, The First Affiliated Hospital, College of Medicine, Zhejiang \\ University, Hangzhou 310003, China; ${ }^{10}$ Department of Gastroenterology, The First Affiliated Hospital of China Medical University, Shenyang \\ 110001, China; ${ }^{11}$ Department of Gastroenterology, Xi'an Central Hospital, Xi'an 710003, China; ${ }^{12}$ Difficult \& Complicated Liver Diseases and \\ Artificial Liver Center, Beijing YouAn Hospital, Capital Medical University, Beijing 100069, China \\ Contributions: (I) Conception and design: X Qi; (II) Administrative support: X Qi; (III) Provision of study materials or patients: All authors; \\ (IV) Collection and assembly of data: Y Wu, Q Zhu, F Meng, S Lin, B Liu, B Li, S Tang, Y Yang, Y Li, S Yuan, Y Chen; (V) Data analysis and \\ interpretation: Y Wu, Q Zhu, F Meng, S Lin, B Liu, B Li, S Tang, Y Yang, Y Li, S Yuan, Y Chen; (VI) Manuscript writing: All authors; (VII) Final \\ approval of manuscript: All authors. \\ \#These authors contributed equally to this work. \\ Correspondence to: Dr. Xingshun Qi, MD. Department of Gastroenterology, General Hospital of Northern Theater Command, No. 83 Wenhua Road, \\ Shenyang, 110840 Liaoning Province, China. Email: xingshunqi@126.com.
}

Background: Our previous study developed Liaoning score as a non-invasive approach for predicting esophageal varices (EVs) in liver cirrhosis. This nationwide multicenter cross-sectional study aimed to externally validate the diagnostic accuracy of Liaoning score and further evaluate its performance for predicting high-risk EVs.

Methods: Cirrhotic patients with acute gastrointestinal bleeding (GIB) without history of endoscopic variceal therapy who underwent endoscopic examinations at their admissions were included. Liaoning score and several non-invasive liver fibrosis scores, including aspartate aminotransferase (AST) to platelet ratio index (APRI), AST to alanine aminotransferase ratio (AAR), fibrosis 4 index (FIB-4), King, and Lok scores, were evaluated. Area under curves (AUCs), cut-off value, sensitivity, and specificity were calculated.

Results: Overall, 612 patients were included. The prevalence of EVs and high-risk EVs was $96.2 \%$ and 95.6\%, respectively. In overall patients, the AUCs of Liaoning score for predicting EVs and high-risk EVs were higher than non-invasive liver fibrosis scores (0.737 versus $0.626-0.721 ; 0.734$ versus $0.611-0.719)$. The cut-off value of Liaoning score for high-risk EVs was 0.477 with a sensitivity of $81.96 \%$ and a specificity of $65.22 \%$. In patients with hematemesis, Liaoning score could significantly predict EVs and high-risk EVs (AUCs $=0.708$ and 0.702, respectively), but not non-invasive liver fibrosis scores. The cut-off value of Liaoning score for high-risk EVs was 0.437 with a sensitivity of $83.16 \%$ and a specificity of $60 \%$.

Conclusions: Liaoning score should be a non-invasive alternative for predicting EVs and high-risk EVs in cirrhotic patients with acute GIB. 
Keywords: Accuracy; endoscopy; esophageal varices (EVs); diagnosis; Liaoning score

Submitted Sep 12, 2019. Accepted for publication Nov 05, 2019.

doi: $10.21037 /$ atm.2019.11.78

View this article at: http://dx.doi.org/10.21037/atm.2019.11.78

\section{Introduction}

Advanced cirrhosis often presents many complications, such as portosystemic collateral vessels, variceal bleeding, ascites, and hepatic encephalopathy (HE) (1). Esophageal varices (EVs) are the most common collateral vessels secondary to portal hypertension in cirrhotic patients and often develop at a rate of $7 \%$ per year (2). Acute gastrointestinal bleeding (GIB) caused by variceal rupture in cirrhotic patients is life-threatening with a high 6-week mortality of $15-25 \%$ $(1,2)$. Considering that endoscopy is often invasive and less available in some remote areas, our previous multicenter observational study conducted in Liaoning province, China established Liaoning score for non-invasively predicting EVs (3), which were based on some simple variables, and found that Liaoning score had a better performance in diagnosing EVs as compared to several other non-invasive scores in patients who had never undergone endoscopy. However, its diagnostic performance for presence of EVs was not externally validated and its performance for predicting high-risk EVs remained unclear.

For this reason, we conducted this present study to validate the diagnostic performance of Liaoning score in a large number of patients from Chinese multi-institutions.

\section{Methods}

Based on the TORCH study, we further screened the eligible patients for the present study. The approval number from the medical ethical committee of our hospital was $\mathrm{k}$ [2019] 21. The inclusion criteria were as follows: (I) cirrhotic patients were diagnosed with acute GIB, which refers to hematemesis and/or melena within 5 days at admission; and (II) endoscopic examinations were performed to evaluate the presence of EVs, regardless of endoscopic therapy. The exclusion criteria were as follows: (I) patients had a history of endoscopic variceal therapy; (II) endoscopic reports were not available or detailed description of EVs was missing; (III) the data regarding Liaoning score were not available; and (IV) the data regarding the characteristics of patients were incomplete.
The data were collected as follows: age, sex, etiology of liver diseases, HE, ascites, red blood cell, hemoglobin, white blood cell, platelet, total bilirubin (TBIL), albumin (ALB), alanine aminotransferase (ALT), aspartate aminotransferase (AST), alkaline phosphatase, $\gamma$-glutamine transferase, blood urea nitrogen, serum creatinine (SCr), prothrombin time, activated partial thromboplastin time, and international normalized ratio (INR).

Child-Pugh (4) and model for end-stage of liver disease (MELD) (5) scores were calculated to evaluate the degree of liver dysfunction.

Child-Pugh score $=$ ALB score + TBIL score + INR score + ascites score $+\mathrm{HE}$ score

MELD score $=9.57 \times \ln [\mathrm{SCr}(\mu \mathrm{mol} / \mathrm{L}) \times 0.011]+3.78 \times$ $\ln [$ TBIL $(\mu \mathrm{mol} / \mathrm{L}) \times 0.058]+11.2 \times \ln (\mathrm{INR})+6.43$

Liaoning score and other non-invasive scores, such as AST to PLT ratio index (APRI) (6), AST to ALT ratio (AAR) (7), fibrosis 4 index (FIB-4) (8), King (9), and Lok (10) score, were also calculated.

Liaoning score for acute GIB $=1.205+1.557 \times$ ascites $(1=$ yes; $0=$ no $)-0.008 \times$ PLT

APRI score $=[($ AST $/$ upper limit of normal $) \times 100] /$ PLT

AAR score $=$ AST $/$ ALT

FIB-4 $=($ age $\times$ AST $) /\left(\mathrm{PLT} \times \mathrm{ALT}^{1 / 2}\right)$

King $=$ age $\times$ AST $\times$ INR/PLT

Lok: $\operatorname{logodds}=-5.56-0.0089 \times \mathrm{PLT}+1.26 \times$ AST $/$ ALT ratio + 5.27 $\times$ INR

Lok $=[\exp (\operatorname{logodds})] /[1+\exp (\operatorname{logodds})]$

The presence of EVs and high-risk EVs were recorded. High-risk EVs were considered, if any one of the following endoscopic features was met: (I) beaded or tumor-like EVs; (II) EVs with red color signs; (III) EVs with clots; or (IV) the maximal diameter of $\mathrm{EVs}$ was $>0.5 \mathrm{~cm}(11,12)$.

\section{Statistical analysis}

The SPSS software version 20.0 (IBM Corp, Armonk, NY, USA) and MedCalc software version 11.4.2.0 (MedCalc Software, Mariakerke, Belgium) were employed to perform all statistical analyses. Continuous variables were described as mean \pm standard deviation and median with range. 
Categorical variables were described as frequencies and percentages. We used receiver operator characteristic (ROC) curves to explore the diagnostic performance of non-invasive scores. Area under curve (AUC), sensitivity, specificity, positive predictive value (PPV), and negative predictive value (NPV) were calculated. The optimal cutoff value of Liaoning score for predicting the presence of EVs obtained from our previous study was 0.485. Its diagnostic performance was confirmed in the present study. We further evaluated the performance of Liaoning score for predicting high-risk EVs. Subgroup analyses were performed in patients with hematemesis. $\mathrm{P}<0.05$ was considered statistically significant.

\section{Results}

\section{Patients}

We totally included 612 cirrhotic patients with acute GIB. Patient characteristics were shown in Table 1. The mean age was $56.08 \pm 12.00$ years. Among them, $73.0 \%$ (447/612) patients were male. The major etiologies of cirrhosis were hepatitis B infection and alcohol abuse $(51.3 \%$ and $26.1 \%$, respectively). Prevalence of EVs and high-risk EVs was 96.2\% (589/612) and 95.6\% (499/522), respectively. In subgroup of patients with hematemesis, prevalence of EVs and high-risk EVs was 96.8\% (453/468) and 96.3\% (386/401), respectively.

\section{Overall analysis}

\section{EVs}

The performance of non-invasive scores for predicting EVs was shown in Table 2.

The AUC of Liaoning score for predicting EVs was 0.737 (95\% CI: $0.700-0.771, \mathrm{P}<0.0001$ ). By comparison, the AUCs of APRI, AAR, FIB-4, King, and Lok scores for predicting EVs were 0.650 (95\% CI: 0.611-0.688, $\mathrm{P}=0.0331), 0.626$ (95\% CI: 0.586-0.664, $\mathrm{P}=0.0330$ ), 0.709 (95\% CI: $0.671-0.745, \mathrm{P}=0.0009), 0.658$ (95\% CI: $0.628-0.695, \mathrm{P}=0.0200)$, and 0.721 (95\% CI: $0.683-0.756$, $\mathrm{P}=0.0004)$, respectively.

Four hundred and ninety-two $(80.4 \%)$ patients had a Liaoning score of greater than 0.485 . Among them, 484 (98.4\%) patients had EVs and 8 (1.6\%) patients did not have EVs. Sensitivity, specificity, PPV, and NPV were $82.17 \%, 65.22 \%, 98.4 \%$, and $12.5 \%$, respectively.

\section{High-risk EVs}

The performance of non-invasive scores for predicting high-risk EVs was shown in Table 2.

The AUC of Liaoning score for predicting high-risk EVs was 0.734 (95\% CI: 0.694-0.771, P=0.0001). By comparison, the AUCs of APRI, AAR, FIB-4, King, and Lok scores for predicting high-risk EVs were 0.647 (95\% CI: 0.604-0.688, $\mathrm{P}=0.0395$ ), 0.611 (95\% CI: 0.568-0.653, $\mathrm{P}=0.0623$ ), 0.703 (95\% CI: 0.661-0.742, $\mathrm{P}=0.0014$ ), 0.654 (95\% CI: $0.611-0.695, \mathrm{P}=0.0246)$, and 0.719 (95\% CI: $0.678-0.757, \mathrm{P}=0.0004)$, respectively.

The optimal cut-off value was 0.477 with a sensitivity, specificity, PPV, and NPV of $81.96 \%, 65.22 \%, 98.1 \%$, and $14.3 \%$, respectively. Four hundred and seventeen (79.9\%) patients had a Liaoning score of greater than 0.477. Among them, 409 (98.1\%) patients had high-risk EVs and 8 (1.9\%) patients did not have high-risk EVs.

\section{Subgroup analysis in patients with hematemesis}

\section{EVs}

The performance of non-invasive scores for predicting EVs in patients with hematemesis was shown in Table 3.

The AUC of Liaoning score for predicting EVs was 0.708 (95\% CI: 0.665-0.749, $\mathrm{P}=0.0016)$. By comparison, the AUCs of APRI, AAR, FIB-4, King, and Lok scores for predicting EVs were 0.585 (95\% CI: 0.539-0.630, $\mathrm{P}=0.3453$ ), 0.602 (95\% CI: 0.556-0.646, $\mathrm{P}=0.1937$ ), 0.609 (95\% CI: 0.563-0.654, $\mathrm{P}=0.1576), 0.603$ (95\% CI: $0.557-0.647, \mathrm{P}=0.1550)$, and 0.549 (95\% CI: 0.502-0.594, $\mathrm{P}=0.5373)$, respectively.

Three hundred and seventy-six $(80.3 \%)$ patients had a Liaoning score of greater than 0.485 . Among them, 370 (98.4\%) patients had EVs and 6 (1.6\%) patients did not have EVs. Sensitivity, specificity, PPV, and NPV were $81.68 \%, 60 \%, 98.4 \%$, and $9.8 \%$, respectively.

\section{High-risk EVs}

The performance of non-invasive scores for predicting high-risk EVs in patients with hematemesis was shown in Table 3.

The AUC of Liaoning score for predicting high-risk EVs was 0.702 (95\% CI: 0.755-0.746, P=0.0147). By comparison, the AUCs of APRI, AAR, FIB-4, King, and Lok scores for predicting high-risk EVs were 0.583 (95\% CI: $0.533-0.632, \mathrm{P}=0.3658), 0.588$ (95\% CI: 0.538-0.637, $\mathrm{P}=0.2630), 0.611$ (95\% CI: 0.561-0.659, $\mathrm{P}=0.1508), 0.605$ 
Table 1 Baseline characteristics of patients

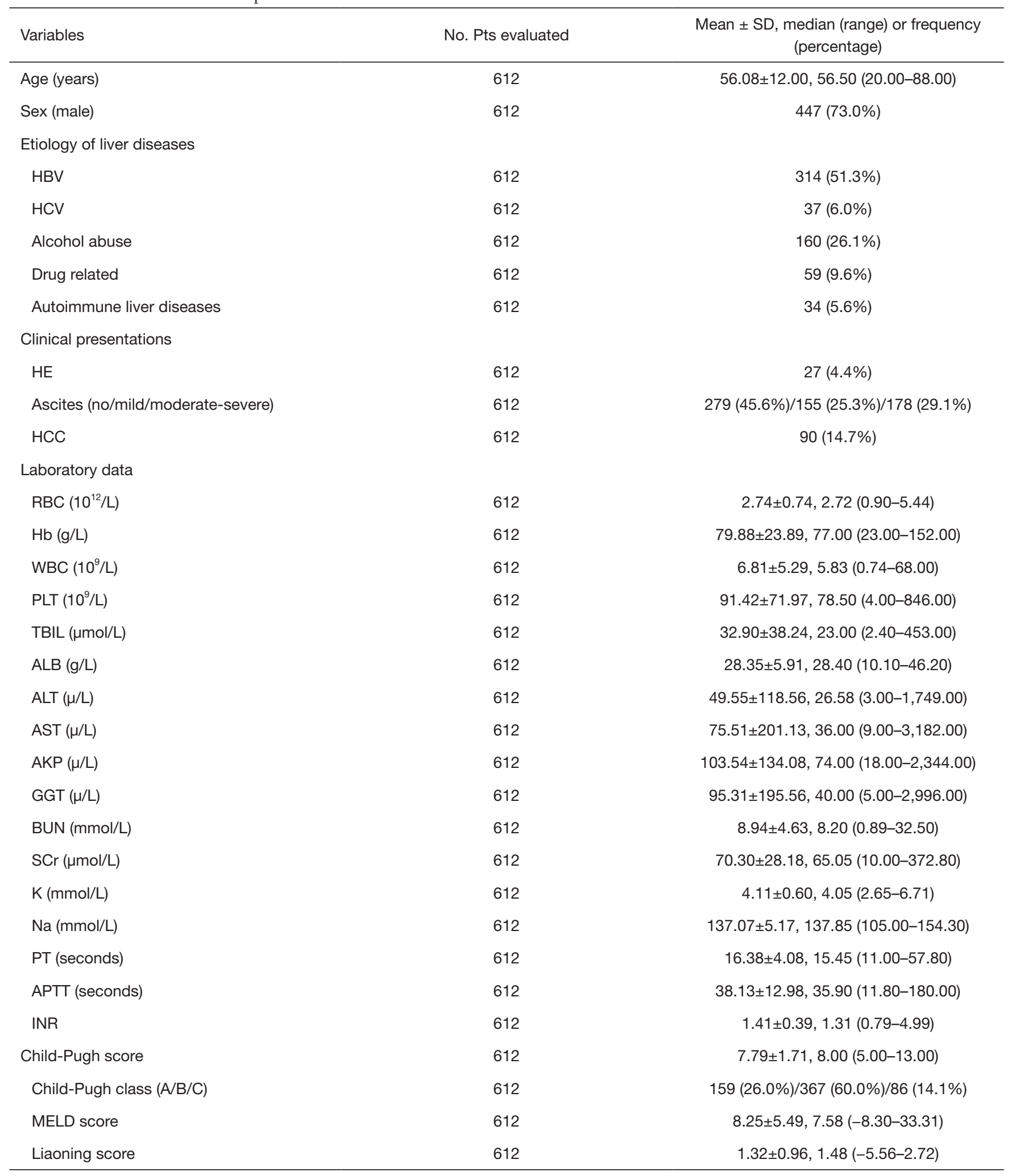

Table 1 (continued) 
Table 1 (continued)

\begin{tabular}{lcr}
\hline Variables & No. Pts evaluated & $\begin{array}{c}\text { Mean } \pm \text { SD, median (range) or frequency } \\
\text { (percentage) }\end{array}$ \\
\hline APRI score & 612 & $1.60 \pm 1.00,1.36(0.08-11.06)$ \\
AAR score & 612 & $3.65 \pm 22.15,1.23(0.07-509.50)$ \\
FIB-4 score & 612 & $8.32 \pm 14.58,5.49(0.42-277.30)$ \\
King score & 612 & $132.07 \pm 916.02,38.73(2.09-20,469.67)$ \\
Lok score & 612 & $0.87 \pm 0.16,0.93(0.02-1.00)$ \\
EVs on endoscopy & 612 & $589(96.2 \%)$ \\
High-risk EVs on endoscopy & 522 & $499(95.6 \%)$ \\
\hline
\end{tabular}

Pts, patients; SD, standard deviation; HBV, hepatitis B virus; HCV, hepatitis C virus; HE, hepatic encephalopathy; HCC, hepatocellular carcinoma; RBC, red blood cell; Hb, hemoglobin; WBC, white blood cell; PLT, platelet; TBIL, total bilirubin; ALB, albumin; ALT, alanine aminotransferase; AST, aspartate aminotransferase; AKP, alkaline phosphatase; GGT, gamma-glutamyl transpeptidase; BUN, blood urea nitrogen; SCr, serum creatinine; K, potassium; Na, sodium; PT, prothrombin time; APTT, activated partial thromboplastin time; INR, international normalized ratio; MELD, model for end-stage of liver disease; APRI, AST to PLT index; AAR, AST to ALT ratio; FIB-4, fibrosis 4 index; EVs, esophageal varices.

(95\% CI: $0.555-0.653, \mathrm{P}=0.1393)$, and 0.550 (95\% CI: $0.500-0.599, \mathrm{P}=0.5291)$, respectively.

The optimal cut-off value was 0.437 with a sensitivity, specificity, PPV, and NPV of $83.16 \%, 60 \%, 98.2 \%$, and $12.2 \%$, respectively. Three hundred and twenty-seven (81.5\%) patients had a Liaoning score of greater than 0.437 . Among them, 321 (98.2\%) patients had high-risk EVs and 6 (1.8\%) patients did not have high-risk EVs.

\section{Discussion}

Based on the Liaoning score that we previously established by using simple laboratory and clinical data (3), the present study aimed to verify the diagnostic accuracy of EVs. We confirmed that Liaoning score could accurately predict the presence of EVs with an optimal cut-off value of 0.485 , and the missing rate was $17.8 \%$, which were similar to our previous study. Because our previous study did not standardize the description of EVs under endoscopy, the performance of Liaoning score for predicting high-risk EVs were not previously evaluated. The present study further found that Liaoning score could accurately predict the presence of high-risk EVs with a cut-off value of 0.477 , and the missing rate was $18 \%$.

Patients with acute upper gastrointestinal bleeding (AUGIB) often present with hematemesis and/or melena (13). Regardless of source of AUGIB, patients with hematemesis have worse prognosis than those with melena alone $(14,15)$. The prognosis of cirrhotic patients with hematemesis secondary to variceal rupture is much worse than those with melena alone (16). Considering the heterogeneity in the treatment selection between patients with variceal and non-variceal bleeding $(11,17,18)$, it is clinically important to identify the presence of varices, especially in patients with hematemesis. Our subgroup analysis of patients with hematemesis showed that Liaoning score was the only non-invasive alternative with a significant diagnostic performance of $\mathrm{EVs}$ and high-risk $\mathrm{EVs}$, but not other non-invasive scores. These findings promote the use of Liaoning score at some hospitals without emergency endoscopy.

Splenomegaly and hypersplenism are often secondary to portal hypertension in liver cirrhosis, which are one of the causes for low PLT (19). PLT was confirmed to be associated with the presence of EVs, but the accuracy of PLT alone for diagnosing EVs was only moderate (20). Portal hypertension is often associated with liver fibrosis. Several non-invasive scores for reflecting the severity of liver fibrosis have been explored to predict EVs. Baveno VI consensus has proposed to spare endoscopy by using PLT count and liver stiffness, which have been verified by several studies with high accuracy $(11,21-23)$. Besides, metaanalyses also found that APRI, AAR, FIB-4, King, and Lok scores for predicting EVs and high-risk EVs were 0.67740.7885 and $0.7095-0.7448$, respectively (24). However, these alternatives had been almost explored in patients with compensated cirrhosis. Notably, the pathophysiology is totally different between compensated and decompensated 


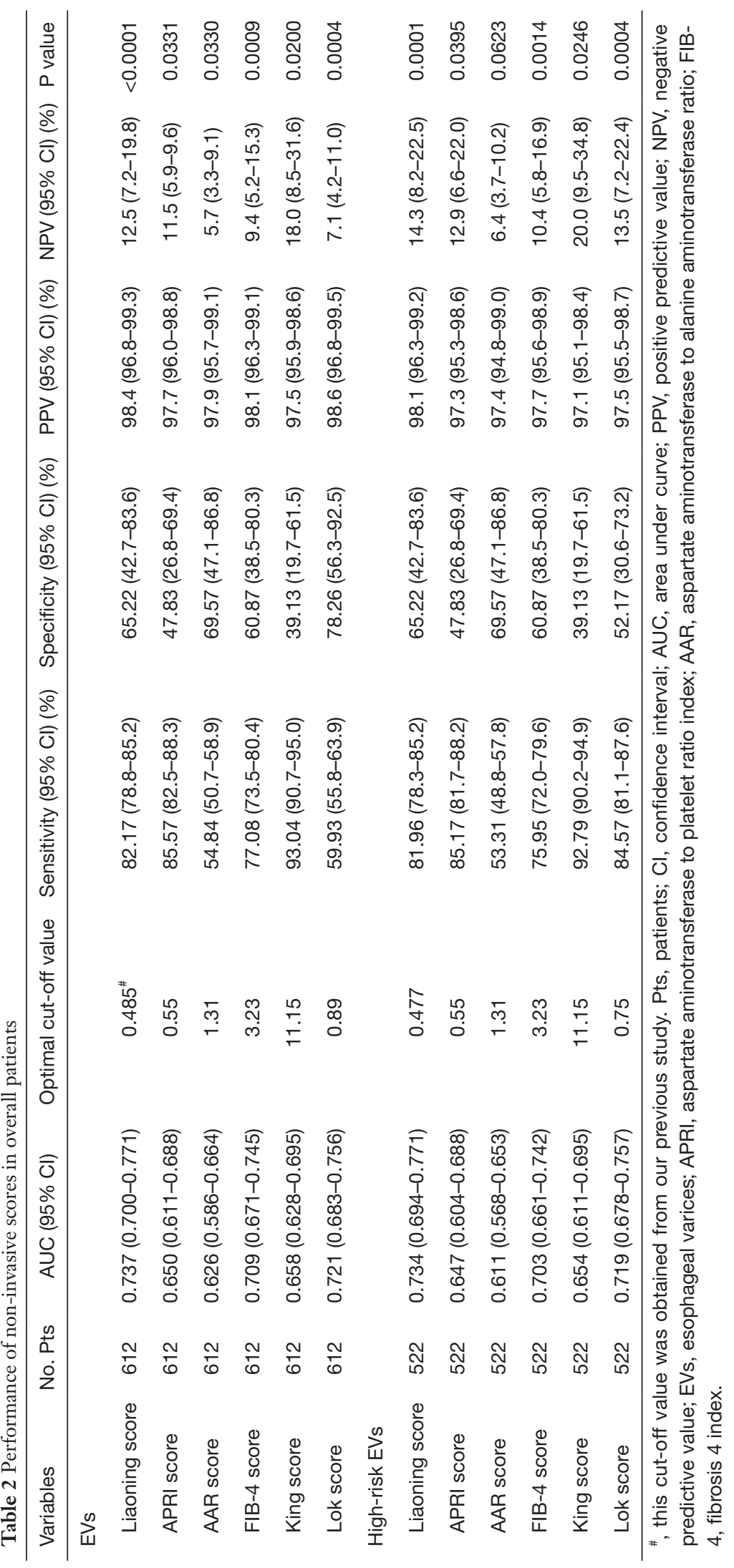




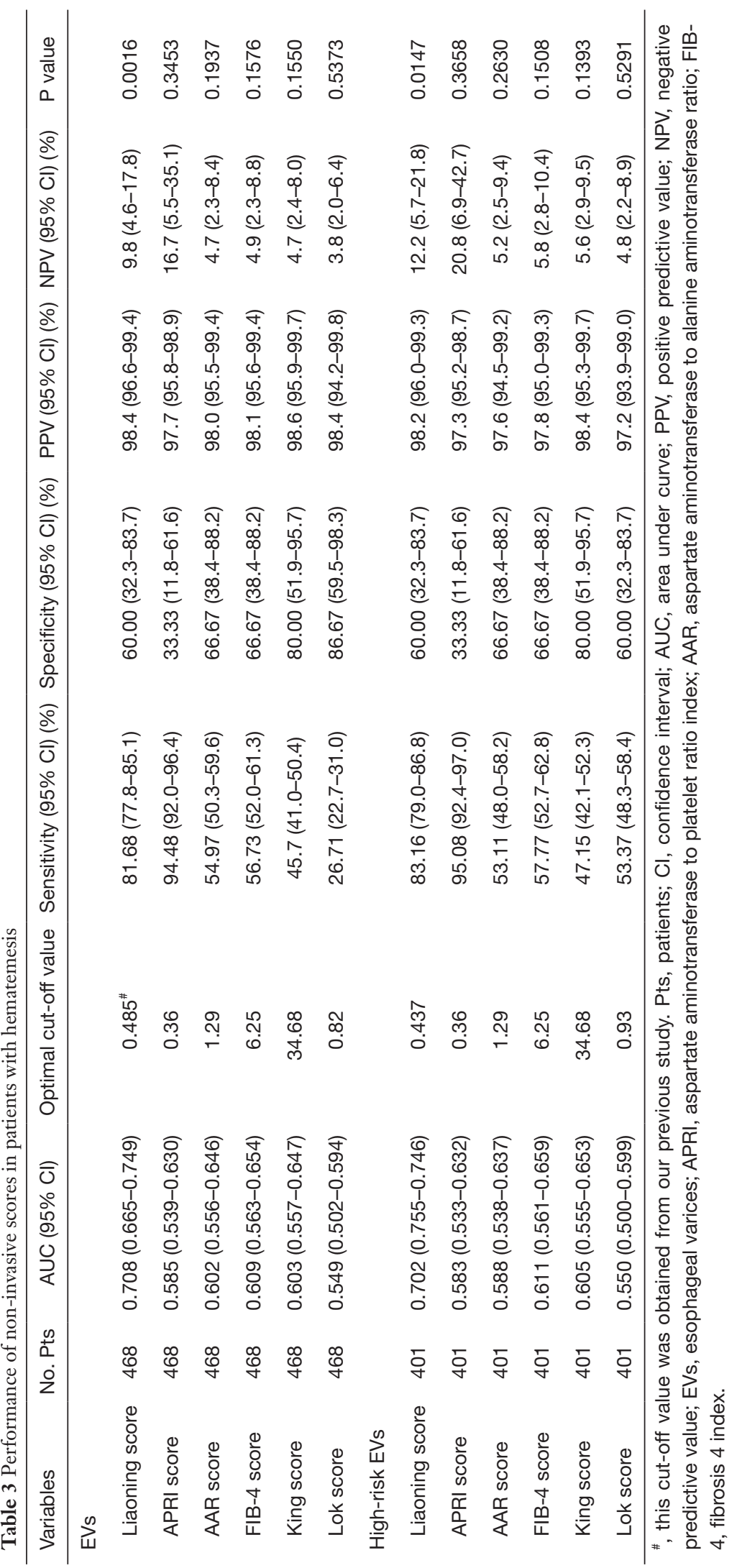


cirrhosis (25). By comparison, all the patients included in the present study were diagnosed as acute GIB and most of them were Child-Pugh B and C. Thus, our present study suggested that these alternatives had slightly lower diagnostic performance (AUCs $=0.626-0.721$ for $\mathrm{EVs}$, and AUCs $=0.611-0.719$ for high-risk EVs). Indeed, Rockey et al. also confirmed that their diagnostic performance were poor in cirrhotic patients with acute GIB (26). Hanafy et al. explored a new scoring system for predicting the presence of EVs, i.e., Glasgow Blatchford score combined with variceal metric score (27). This scoring system was complex and its components were not easy to access, despite it could obtain a good performance with an AUC of 0.989 in validation cohort. By comparison, Liaoning score is easier to be calculated.

There were several limitations in our study. First, the bias in selection of patients could not be inevitable among the participating centers. Second, the prevalence of EVs and high-risk EVs was high, which led to a low NPV. Thus, we could not calculate the rate of spared endoscopy. Third, the TORCH study enrolled cirrhotic patients with acute GIB alone, so we could not verify the diagnostic performance of Liaoning score in patients without acute GIB. Fourth, hepatic venous pressure gradient measurement can directly reflect the degree of portal hypertension, but it is invasive and expensive and requires technical skill. It was not regularly performed in our patients, especially when they presented with acute bleeding episodes. Fifth, we used the old version of MELD score formula in the present study and some patients had negative scores. However, this behavior did not influence its prognostic impact.

In conclusion, Liaoning score could be considered for predicting EVs and high-risk EVs in cirrhotic patient with acute GIB, which might be useful for identifying the source of GIB and guiding treatment selection.

\section{Acknowledgments}

None.

\section{Footnote}

Conflicts of Interest: The authors have no conflicts of interest to declare.

Ethical Statement: The authors are accountable for all aspects of the work in ensuring that questions related to the accuracy or integrity of any part of the work are appropriately investigated and resolved. The approval number from the medical ethical committee of our hospital was k [2019] 21.

\section{References}

1. Tsochatzis EA, Bosch J, Burroughs AK. Liver cirrhosis. Lancet 2014;383:1749-61.

2. Chakinala RC, Kumar A, Barsa JE, et al. Downhill esophageal varices: a therapeutic dilemma. Ann Transl Med 2018;6:463.

3. Qi X, Li Y, Wang R, et al. Liaoning Score for Prediction of Esophageal Varices in Cirrhotic Patients Who Had Never Undergone Endoscopy: A Multicenter CrossSectional Study in Liaoning Province, China. Adv Ther 2019;36:2167-78.

4. Pugh RN, Murray-Lyon IM, Dawson JL, et al. Transection of the oesophagus for bleeding oesophageal varices. $\mathrm{Br} \mathrm{J}$ Surg 1973;60:646-9.

5. Kamath PS, Kim WR. The model for end-stage liver disease (MELD). Hepatology 2007;45:797-805.

6. Wai CT, Greenson JK, Fontana RJ, et al. A simple noninvasive index can predict both significant fibrosis and cirrhosis in patients with chronic hepatitis C. Hepatology 2003;38:518-26.

7. Giannini E, Risso D, Botta F, et al. Validity and clinical utility of the aspartate aminotransferase-alanine aminotransferase ratio in assessing disease severity and prognosis in patients with hepatitis $\mathrm{C}$ virus-related chronic liver disease. Arch Intern Med 2003;163:218-24.

8. Sterling RK, Lissen E, Clumeck N, et al. Development of a simple noninvasive index to predict significant fibrosis in patients with HIV/HCV coinfection. Hepatology 2006;43:1317-25.

9. Cross TJ, Rizzi P, Berry PA, et al. King's Score: an accurate marker of cirrhosis in chronic hepatitis C. Eur J Gastroenterol Hepatol 2009;21:730-8.

10. Lok AS, Ghany MG, Goodman ZD, et al. Predicting cirrhosis in patients with hepatitis $\mathrm{C}$ based on standard laboratory tests: results of the HALT-C cohort. Hepatology 2005;42:282-92.

11. de Franchis R, Baveno VIF. Expanding consensus in portal hypertension: Report of the Baveno VI Consensus Workshop: Stratifying risk and individualizing care for portal hypertension. J Hepatol 2015;63:743-52.

12. Chinese Society of Gastroenterology $\mathrm{CSoH}$, Chinese Society of Endoscopy, Chinese Medical Association. Consensus on prevention and treatment for 
gastroesophageal varices and variceal hemorrhage in liver cirrhosis. Chinese Journal of Digestion 2008;28:551-8.

13. Meltzer AC, Klein JC. Upper gastrointestinal bleeding: patient presentation, risk stratification, and early management. Gastroenterol Clin North Am 2014;43:665-75.

14. Laine L, Laursen SB, Zakko L, et al. Severity and Outcomes of Upper Gastrointestinal Bleeding With Bloody Vs. Coffee-Grounds Hematemesis. Am J Gastroenterol 2018;113:358-66.

15. Kim JJ, Sheibani S, Park S, et al. Causes of bleeding and outcomes in patients hospitalized with upper gastrointestinal bleeding. J Clin Gastroenterol 2014;48:113-8.

16. Chen PH, Chen WC, Hou MC, et al. Delayed endoscopy increases re-bleeding and mortality in patients with hematemesis and active esophageal variceal bleeding: a cohort study. J Hepatol 2012;57:1207-13.

17. Garcia-Tsao G, Abraldes J, Berzigotti A, et al. Portal hypertensive bleeding in cirrhosis: Risk stratification, diagnosis, and management: 2016 practice guidance by the American Association for the study of liver diseases. Hepatology 2017;65:310-35.

18. Sung JJ, Chiu PW, Chan FKL, et al. Asia-Pacific working group consensus on non-variceal upper gastrointestinal bleeding: an update 2018. Gut 2018;67:1757-68.

19. Peck-Radosavljevic M. Thrombocytopenia in chronic liver disease. Liver Int 2017;37:778-93.

20. Abd-Elsalam S, Habba E, Elkhalawany W, et al. Correlation of platelets count with endoscopic findings in a cohort of Egyptian patients with liver cirrhosis. Medicine (Baltimore) 2016;95:e3853.

21. Maurice JB, Brodkin E, Arnold F, et al. Validation of the Baveno VI criteria to identify low risk cirrhotic patients not requiring endoscopic surveillance for varices. J Hepatol 2016;65:899-905.

22. Augustin S, Pons M, Maurice JB, et al. Expanding the Baveno VI criteria for the screening of varices in patients with compensated advanced chronic liver disease. Hepatology 2017;66:1980-8.

23. Bae J, Sinn DH, Kang W, et al. Validation of the Baveno VI and the expanded Baveno VI criteria to identify patients who could avoid screening endoscopy. Liver Int 2018;38:1442-8.

24. Deng H, Qi X, Guo X. Diagnostic Accuracy of APRI, AAR, FIB-4, FI, King, Lok, Forns, and FibroIndex Scores in Predicting the Presence of Esophageal Varices in Liver Cirrhosis: A Systematic Review and Meta-Analysis. Medicine (Baltimore) 2015;94:e1795.

25. Jakab SS, Garcia-Tsao G. Screening and Surveillance of Varices in Patients With Cirrhosis. Clin Gastroenterol Hepatol 2019;17:26-9.

26. Rockey DC, Elliott A, Lyles T. Prediction of esophageal varices and variceal hemorrhage in patients with acute upper gastrointestinal bleeding. J Investig Med 2016;64:745-51.

27. Hanafy AS, Badawi R, Basha MAA, et al. A novel scoring system for prediction of esophageal varices in critically ill patients. Clin Exp Gastroenterol 2017;10:315-25.
Cite this article as: $\mathrm{Li} \mathrm{Q}, \mathrm{Wu} \mathrm{Y,} \mathrm{Zhu} \mathrm{Q,} \mathrm{Meng} \mathrm{F,} \mathrm{Lin} \mathrm{S,} \mathrm{Liu}$ B, Li B, Tang S, Yang Y, Li Y, Yuan S, Chen Y, Qi X. External validation of Liaoning score for predicting esophageal varices in liver cirrhosis: a Chinese multicenter cross-sectional study. Ann Transl Med 2019;7(23):755. doi: 10.21037/atm.2019.11.78 